\title{
Validación de encuesta de percepción Examen Nacional de Enfermería Chileno
}

\section{Validation of perception survey Chilean National Nursing Exam}

\section{Pesquisa de validação da percepção Exame Nacional de Enfermagem do Chile}

\section{Verónica Marcela Molina-Rodríguez'; María Patricia Masalan-Apip²; Verónica Del Carmen Drago-Machado3; Gloria Alejandra García-Vallejos ${ }^{4}$; Irella Alejandra Vergara-Beltrán ${ }^{5}$ \& Alejandra F. Rojas-Rivera ${ }^{6}$}

${ }^{1}$ Enfermera. Magíster en Pedagogía Universitaria. Profesor Asistente, Directora Docente, Escuela de Enfermería de la Universidad Mayor, sede Santiago, Chile. Orcid: 0000-0002-0360-9458. Correo electrónico: veronica.molina@umayor.cl

${ }^{2}$ Enfermera Matrona. Magister en Salud Pública. Profesor Asociado, Directora de Extensión y Educación Continua, Escuela de Enfermería de la Pontificia Universidad Católica de Chile. Orcid:0000-0001-7723-803. Correo electrónico: mmasalan@uc.cl

${ }^{3}$ Enfermera. Diplomada en Pedagogía Universitaria. Profesor Asistente, Escuela de Enfermería de la Universidad Católica de la Santísima Concepción, Chile. Orcid:0000-0001-5398-3686. Correo electrónico: vdrago@ucsc.cl

${ }^{4}$ Enfermera. Magíster en Psicología Mención Psicología Comunitaria. Profesor Asistente, Escuela de Enfermería de la Universidad de Chile. Orcid: 0000-0001-7173-6179. Correo electrónico: ggarcia@med.uchile.cl

${ }^{5}$ Enfermera. Magíster en Educación en Ciencias de la Salud. Profesor Asistente, Secretaria Académica, Escuela de Enfermería de la Universidad Diego Portales, Chile. Orcid: 0000-0002-5976-2056. Correo electrónico: irella.vergara@udp.cl

${ }^{6}$ Enfermera. Magíster en Gerontología Social. Profesor Asociado, Directora de Pos títulos de la Escuela de Enfermería de la Universidad de los Andes, Chile. Orcid:0000-0001-5134-4135. Correo electrónico: arojas@uandes.cl

Correspondencia: Pontificia Universidad Católica de Chile. Avenida Vicuña Mackenna No. 4860. Macul. Santiago de Chile.

Correo electrónico contacto: mmasalan@uc.cl

Cómo citar este artículo: Molina Rodríguez, V.M., Masalan Apip, M.P., Drago Machado V.C., García Vallejos, G.A., Vergara Beltan, I.A., \& Rojas. Rivera, A.F. (2021). Validación de encuesta de percepción Examen Nacional de Enfermería Chileno. Cultura de los Cuidados (Edición digital), 25(59). Recuperado de http://dx.doi.org/10.14198/cuid.2021.59.16

Recibido:01/11/2020 Aceptado: 13/01/2021

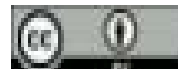




\section{RESUMEN}

Investigación descriptiva cuantitativa y cualitativa. Objetivo: validar encuesta de percepción de los egresados de Enfermería en Chile, respecto al Examen Nacional de Enfermería. Metodología: lo cualitativo consideró el método Delphi participando expertos en la disciplina de enfermería y del área educación y evaluación. Para lo cuantitativo se utilizó el análisis de alfa de Cronbach, usando el sistema SPSS. Resultados: El K obtenido por los expertos estuvo entre el 0,95 a 1 lo que determinó que todos tenían un alto grado de competencia en el tema sobre el cual se les consultó. Los expertos vincularon los objetivos propuestos con cada uno de los 21 ítems de la encuesta. En 8 de ellos el 100\% de los expertos coincidió con lo plateado por la Comisión del Examen Nacional de Enfermería, en 10 de los ítems un 80\%, en 2 de los ítems un 50\% y en 1 ítem un 33\%. El alpha de Cronbach obtenido fue de 0,97, lo que demuestra un alto grado de consistencia interna del instrumento. Conclusiones: Se valida la encuesta de opinión, cumpliendo de manera rigurosa y lógica con todas sus etapas, demostrando su validez $\mathrm{y}$ confiabilidad.

Palabras clave: Educación en enfermería; instrumento de evaluación; encuestas; confiabilidad; validez.

\section{ABSTRACT}

Quantitative and qualitative descriptive research. Objective: to validate the survey on the perception of nursing graduates in Chile, regarding the National Exam taken and applied. Methodology: qualitative, using the Delphi method with experts in the areas of nursing, education, and evaluation; and quantitative, using Cronbach's alpha analysis with the SPSS system. Results: The $\mathrm{K}$ was between 0.95 to
1 , which suggests that all of the experts had a high degree of competence in the opinion topic. The objectives proposed in the 21item survey were linked. In 8 items $100 \%$ of the experts stated their agreement with the aspects raised by the National Nursing Exam Commission; in 10 items $80 \%$, in 2 items $50 \%$ and in 1 item 33\%. Cronbach's alpha method estimated the internal consistency reliability of the instrument to be 0.97 , demonstrating a high degree of internal consistency among the items analyzed. Conclusions: to validate an instrument it is necessary to rigorously complete each step with a sequence that allows one to account for validity and reliability.

Keywords: Nursing education; assessment instrument; surveys; reliability; validity

\section{RESUMO}

Quantitative and qualitative descriptive research. Objective: to validate the perception survey of nursing graduates in Chile, regarding the National Nursing Exam. Methodology: the Delphi method was considered qualitative, involving experts in the nursing discipline and in the education and evaluation area. For the quantitative analysis, Cronbach's alpha was used, using the SPSS system. Results: The K obtained by the experts was between 0.95 to 1 , which determined that all of them had a high degree of competence in the subject on which they were consulted. The experts linked the proposed objectives with each of the 21 items in the survey. In 8 of them, 100\% of the experts agreed with what was stated by the National Nursing Exam Commission, in 10 of the items $80 \%$, in 2 of the items $50 \%$ and in 1 item 33\%. The Cronbach's alpha obtained was 0.97 , which shows a high degree of internal consistency of the instrument. Conclusions: The opinion poll is validated, rigorously and logically complying with all its stages, demonstrating its validity and reliability. 
Palavras chaves: Educação em enfermagem; instrumento de avaliação; pesquisas; confiabilidade; validade.

\section{INTRODUCCIÓN}

En el año 1963 se crea la Asociación Chilena de Educación en Enfermería (ACHIEEN), corporación de derecho privado, de carácter científico, sin fines de lucro, que tiene como misión velar por la calidad de la formación de las enfermeras y enfermeros en el ámbito de la educación superior. Este organismo cuenta con varias comisiones de trabajo, entre ellas la Comisión de Examen Nacional de Enfermería (CO-ENENF), encargada de desarrollar un examen de selección única, con la asesoría de un organismo experto en evaluación y medición, reconocido a nivel nacional. Este examen es de carácter voluntario para los estudiantes que cursan el último año de la carrera de universidades públicas y privadas a nivel nacional, cuyo propósito es evaluar los conocimientos de los egresados en áreas de la enfermería previamente determinadas (ACHIEEN, 2019).

\section{Objetivo}

El objetivo de este estudio es validar la encuesta utilizada para recoger la percepción de los egresados de enfermería respecto del examen nacional de enfermería y su aplicación.

Desde el año 2013, inmediatamente finalizado el examen, se solicita a los estudiantes contestar una encuesta, cuyo objetivo es conocer su percepción respecto de la prueba y del proceso rendido. Esta encuesta fue diseñada de manera rigurosa por los integrantes de la CO-ENENF con el organismo asesor en evaluación.El estudiante sabe con antelación que al finalizar la aplicación del examen se le pedirá su opinión a través de una encuesta, la cual se aplica de forma presencial en formato papel-lápiz, reguardándose anonimato y confidencialidad. Su confección consideró detalles como la redacción de las aseveraciones, la sintaxis y la semántica en la construcción de las oraciones (Cardenas, 2010).

La encuesta se divide en dos partes: (i) una carta que contiene un formato de consentimiento informado, donde se explicita el propósito de su administración, el ámbito de dominio, el modo cómo serán tratados los datos y la aplicación de los mismos. Incluye también, una declaración voluntaria que debe ser firmada por el encuestado, en que acepta las condiciones de uso de los resultados obtenidos en la encuesta, y (ii) un cuestionario de 21 ítems con 4 opciones de valoración en escala de Likert, 1: muy de acuerdo, 2: de acuerdo, 3 : en desacuerdo, 4: muy en desacuerdo y se incluye la opción no aplica. En cuanto al orden de los ítems, se dispuso lo siguiente: primero se incluyeron los aspectos generales, luego la representatividad $\mathrm{y}$ dominio de las áreas, y por último la utilidad del examen. Los datos obtenidos en esta encuesta durante los años han permitido optimizar aspectos administrativos en la aplicación de este y a su vez mejorar el contenido y orientación de las preguntas de la prueba.

Es preciso señalar que la evaluación es un método intencionado, funcional, continuo e integral para adquirir información de los procesos educativos. De esta forma se puede evidenciar la calidad y mejorar la eficacia de estos procesos (Romero y Espinoza, 2010).

Según Hernandez, Illesca y Cabezas (2013), es relevante conocer la opinión de los estudiantes, ya que es otro factor más del proceso evaluativo, llegando a convertirse en un agente que influye en el ámbito formativo, que permite hacer un aporte al mejoramiento continuo, convirtiéndose en 
Revista científica de la Asociación de Historia y Antropología de los Cuidados (Universidad de Alicante)

una fuente de información básica en la evaluación continua de una institución educativa (Ortiz y Vergel, 2013).

La Real Academia de la Lengua Española, RAE, define la palabra "opinión" como "Un conjunto de preguntas tipificadas dirigidas a una muestra representativa de grupos sociales, para averiguar estados de opinión o conocer otras cuestiones que les afectan”

Según Huneeus (1999), las encuestas de opinión son instrumentos de investigación social y de acuerdo a Cordero y Tapia (2007), en los últimos 30 años se han masificado en todos los sectores productivos y de servicios a nivel mundial. Para Martinez (2008), estas encuestas están sustentadas en objetivos y finalmente Paniagua (2015), plantea que sus preguntas deben tener relación con ellos. Es relevante señalar que para construir una encuesta de opinión se deben considerar diferentes factores y la confección del diseño debe ser rigurosa, cuidando detalles como el orden lógico en la redacción de las preguntas, la sintaxis y la semántica en la confección de las oraciones, el orden en que se disponen en el instrumento y la idoneidad de las preguntas, entre otros (Cardenas, 2010). Para el análisis de los resultados es necesario tener en cuenta la calidad de las respuestas del entrevistado, entre las que se pueden encontrar respuestas poco fiables, equivocaciones, mentiras $\mathrm{y}$ sesgos. Para reducir los errores se pueden utilizar algunas estrategias como: garantizar el anonimato y confidencialidad, utilizar un manual de procedimiento, aumentar el contacto con los entrevistados previo y durante la intervención (Martínez, 2008).
Para

realización

de

investigaciones en educación es importante contar con instrumentos validados y confiables. Según Paniagua (2015), la validez se refiere a que los instrumentos midan lo que se desea medir. Se han definido diferentes tipos de validez entre los que destacan: de contenido, de criterio y de constructo (Leyva, 2011; Bojórquez, López, Hernández y Jiménez, 2013).

Se define validez de contenido al grado en que el instrumento representa la totalidad del fenómeno que se pretende medir, lo que está en directa relación con la calidad del instrumento. Es en este tipo de validez, donde se les solicita a los expertos expresar su opinión en el área, emitiendo un juicio. Es muy relevante realizar esta etapa en forma rigurosa, tanto en la metodología como en el análisis de los datos (Cordero y Tapia, 2007; Escobar y Cuervo, 2008; Galicia, Balderrama y Navarro, 2017; Urrutia, Barrios, Gutiérrez y Mayorga 2014). Para cumplir esta validación se puede emplear el método Delphi que consiste en la utilización sistemática del juicio intuitivo de un grupo de expertos para obtener un consenso de opiniones informadas, de ahí, que también se conoce con el nombre de criterio de expertos. Es uno de los procesos subjetivos de pronóstico más confiable y constituye un procedimiento para confeccionar un cuadro de la evolución de situaciones complejas, mediante la elaboración estadística de las opiniones de los expertos en el tema de que se trate (Von der Gracht, 2012).

El método, se basa en la organización de un diálogo anónimo entre los expertos consultados de modo individual a partir de la aplicación de un cuestionario. Los expertos, seleccionados previamente, se someten a una serie de interrogantes sucesivas, cuyas respuestas se analizan para 
conocer la coincidencia o discrepancia que estos tienen en cuanto a lo consultado (Torrecilla-Salinas, De Troyer, Escalona y Mejías, 2017). Para la aplicación de este método se debe tener presente, la elaboración del cuestionario y la selección del panel de expertos. Para Reguant y Torrado (2016), el experto, es un individuo preparado para opinar respecto a un tema en particular y hacer comentarios y/o sugerencias. De manera que la información recogida sea de calidad, los expertos deben ser competentes, para lo cual se les solicita su autovaloración para finalmente determinar el grado de competencia que tienen respecto al tema consultado. Otros puntos relevantes son la disponibilidad de participar e incluir especialistas de distintas profesiones vinculadas al tema en cuestión.

La validez de criterio está determinada por la comparación entre la ejecución observada y la ejecución esperada. Asimismo, mide la relación entre una variable externa, un índice o un indicador del elemento central que se está evaluando y además del tipo de instrumento que se está utilizando (Guirao-Goris, Ferrer y Montejano, 2016). Por último, la validez de constructo es uno de los principales procesos en la validación, ya que de ésta depende la capacidad de discriminar o distinguir el grado de consecución de la competencia evaluada, lo que garantiza la existencia del constructo en cuestión. Esta es una validación que requiere análisis estadístico (Leyva, 2011). Para Gonzalez (2017), la confiabilidad se refiere a tener ausencia de errores en la medida, siendo determinada por la consistencia, la estabilidad y la reproductibilidad de los resultados similares en condiciones comparables. La importancia de asegurar la confiabilidad radica en minimizar la influencia que tiene el observador, que es quien evalúa y de esta manera reducir los sesgos de medición (Manterola et al., 2018). El Alfa de Cronbach es el indicador de confiabilidad de escalas psicométricas, un coeficiente que se basa en el promedio entre las variables que forman parte de la escala, lo que aporta a la homogeneidad de la misma y cuanto más se aproxime a su valor máximo, 1 , mayor es la fiabilidad de la escala. Además, en determinados contextos y por tácito convenio, se considera que valores del alfa superiores a 0,7 son suficientes para garantizar la fiabilidad de la escala (Cervantes, 2005).

\section{METODOLOGIA}

Se trata de un estudio descriptivo, el cual fue aprobado por el Comité Ético de una de las Universidades chilenas socias de ACHIEEN. Para el proceso de validación de esta encuesta, se utilizaron metodologías cualitativa y cuantitativa.

ETAPA I: Con relación a la metodología cualitativa se consideró el apoyo de expertos basado en el método Delphi. Este proceso consideró los siguientes pasos:

1. Se eligió un panel de expertos. Se seleccionaron 3 docentes de la disciplina de enfermería y 3 especialistas en el área de educación y evaluación. Los docentes de la disciplina corresponden a Enfermeras que habían formado parte de la CO-ENENF en comisiones previas. Los expertos del área de educación y evaluación se desempeñan en Oficinas de Educación Médica de Universidades chilenas pertenecientes a ACHIEEN.

2. Se determinó el grado de competencia de los expertos a través del cálculo del Coeficiente $\mathbf{K}$, el 
Revista científica de la Asociación de Historia y Antropología de los Cuidados (Universidad de Alicante)

cual se obtuvo aplicando la siguiente formula:

$K=\frac{1}{2}\left(K_{c}+K_{a}\right)$

Para Kc, Coeficiente de conocimiento o información que posee la persona, se les solicitó a los expertos que realizaran una autovaloración respecto de la información que manejan sobre el tema. Se utilizó una escala de 0 a 10, donde "0" indica que no posee absolutamente ningún conocimiento de la problemática, mientras que el 10 expresa pleno conocimiento. Dicha nota se multiplica por 0.1 .

Para el cálculo del Ka, Coeficiente de argumentación, se les solicitó a los expertos, identificar el grado de influencia (alto, medio y bajo) que cada una de las fuentes de argumentación tuvieron en sus respuestas. Las fuentes que se consideraron fueron: análisis teóricos realizados, experiencia en el tema, trabajos de autores nacionales, trabajos de autores extranjeros, su propio conocimiento del estado del problema y su intuición. Cada una de estas categorías (alto, medio o bajo) tenía un valor numérico distinto según la fuente (Anexo 1)

A partir de la suma de los valores numéricos de cada fuente se obtiene un puntaje, que corresponde al Ka. Este, representa el grado de influencia que tuvieron todas las fuentes, en las respuestas expresadas por el experto. Ka está definido según el siguiente rango: si es menor de 0.6 se determina como bajo, entre 0.6 y 0.8 el grado es medio y mayor a 0.8 se considera alto.

Una vez calculados Kc y Ka, se aplica la formula descrita anteriormente, para obtener $\mathrm{K}$. Este puede estar entre 0,25 y 1 . Mientras más cercano a 1 , mayor grado de competencia del experto sobre el tema presentado.

3. A los expertos se les envió la encuesta aplicada a los estudiantes el año 2017, junto a un cuestionario tipo, para recoger sus opiniones respecto a dicha encuesta y obtener información de carácter estadístico. El cuestionario consideró los siguientes puntos:

a) Aspecto formal de cada ítem: vocabulario, claridad en la formulación de los ítems, extensión, tiempos de aplicación o cualquier otro aspecto que merezca comentarios o reparos.

b) Coherencia entre cada ítem del instrumento con los objetivos específicos planteados para la encuesta. Para ello, se les solicitó anotar el número del objetivo al cual creía que apuntaba, cada uno de los ítems. Estos eran:

1. Identificar la percepción de los estudiantes de enfermería acerca de aspectos generales del ENENF.

2. Describir la percepción de los estudiantes de enfermería en relación a las áreas que son representativas del quehacer profesional.

3. Identificar la percepción de los estudiantes de enfermería acerca del dominio teórico de las áreas que son representativas del quehacer profesional.

4. Reconocer la percepción de los estudiantes de enfermería respecto a la utilidad del examen como un instrumento para evaluar la calidad en la formación del futuro profesional. 
Posteriormente se analizaron las observaciones de los expertos y se realizaron ajustes a la encuesta base.

ETAPA II: La encuesta base modificada según observaciones de expertos fue aplicada a los estudiantes en la versión del Examen Nacional de Enfermería el año 2018. De un total de 2.978 encuestas, se tomaron al azar 600 para ser analizadas desde la perspectiva del Alpha de Cronbach. Para el análisis de los datos y determinación del Alpha, se utilizó el sistema SPSS (Versión 12.0).

\section{RESULTADOS}

Respecto al nivel de competencia de los expertos se obtuvo, que:

Todos se autoevaluaron con nota de 10, por lo que cada uno obtuvo un Kc de 1

Los Ka estuvieron entre 0,9 a 1, es decir las fuentes de argumentación utilizadas tuvieron altos grados de influencia.

Se obtuvieron K entre 0,95 a 1, lo que indica que todos los expertos tenían un alto grado de competencia en el tema sobre el cual opinaron.

Los comentarios y/o sugerencias realizadas por los expertos, fueron:

- Cambiar el verbo en un ítem.

- Cambiar algunos ítems a afirmaciones en vez de suposiciones

- Dar énfasis en el "cuidado de enfermería" y no en los problemas de salud.

- Replantear uno de los ítems que aparece de manera general.

- Separar en dos uno de los ítemes, de manera de evaluar por separado las dos partes que conforman el examen.

- Especificar en los ítems que hacen mención al dominio teórico, si el estudiante considera contar con este dominio.

- Precisar en el ítem de gestión y liderazgo, si esos conocimientos son los que debería tener un egresado de enfermería.

- Agregar en uno de los ítems la etiqueta conocimiento de más relevancia o fundamentales, de manera de circunscribir un poco más la aseveración.

- Revisar el orden de los ítems con la finalidad de facilitar la fluidez en la respuesta, se podrían posicionar primero todos los que apuntan a aspectos generales del examen.

- Agregar, una pregunta abierta que recoja sugerencias respecto a la realización del examen en futuras versiones.

- $\quad$ "Los ítems tienen una lógica clara y coherente, además de abordar ámbitos relacionados con el estudio y suficientes en cantidad".

- "Los ítems son claros, poseen un vocabulario atingente y una extensión adecuada”.

Posteriormente, los expertos vincularon los objetivos propuestos con cada ítem de la encuesta. De los 21 ítems, en 8 de ellos el $100 \%$ de los expertos coincidió con la comisión, en 10 de los ítems un 80\%, en 2 de los ítems un 50\% y en 1 ítem un 33\%. Ninguno de los expertos sugirió sacar ni agregar algún ítem.

Debido a las sugerencias de los expertos y el análisis de los integrantes de la CO-ENENF, se realizaron ajustes formales y se modificó 
Revista científica de la Asociación de Historia y Antropología de los Cuidados (Universidad de Alicante)

el instrumento dejando 23 ítems en lugar de 21, de manera de optimizar la encuesta, la cual se aplicó en el siguiente versión del examen (Anexo 2). A esta nueva versión se le aplicó Alpha de Cronbach obteniéndose un valor de 0.97 , lo que evidencia un grado mayor de consistencia interna en los ítems analizados.

\section{CONCLUSIÓN}

La validación de un instrumento no es un acto sencillo, requiere del cumplimiento de varias etapas con una secuencia lógica de manera tal que permita dar cuenta de la validez y confiabilidad para que ese instrumento sea utilizado en una investigación.

Cabe destacar, que a la fecha no se contaba en Chile con un instrumento validado que permitiese recoger la percepción de los egresados que rinden el Examen Nacional de Enfermería.

El método de evaluación cuantitativo, utilizado en la investigación, demostró que la encuesta estaba bien construida, lo que concuerda con la evaluación cualitativa, donde los expertos emitieron sugerencias de forma, pero no de fondo, las que se consideraron para la versión definitiva.

Finalmente, se puede señalar que la encuesta validada permite conocer la percepción de los estudiantes respecto al Examen Nacional de Enfermería rendido y el desarrollo del proceso de aplicación, por tanto, es recomendable su aplicación en actividades similares.

\section{BIBLIOGRAFÍA}

Asociacion Chilena de Educacion en Enfermeria ACHIEEN (2019). Recuperado de https://www.achieen.org/ , 2019 Agosto 21

Bojórquez, J., López, L., Hernández, M., y Jiménez E. (agosto 2013). Utilización del alfa de Cronbach para validar la confiabilidad de un instrumento de medición de satisfacción del estudiante en el uso del software Minitab. Eleventh LACCEI Latin American and Caribbean Conference for Engineering and Technology. Innovation in Engineering, Technology and Education for Competitiveness and Prosperity. Cancun, Mexico. Recuperado de http://laccei.org/LACCEI2013-

Cancun/RefereedPapers/RP065.pdf

Cárdenas, V. (2010). La relación entre semántica y sintaxis desde la perspectiva de la producción de lenguaje escrito. Tópicos del seminario, (23), 241289, recuperado de http://www.redalyc.org/articulo.oa?=594/59 415631008

(2005). Interpretaciones del coeficiente de alpha de Cronbach. Avances en Medicion. 3(1), 9-25.

Cordero, R., y Tapia, G. (2007). Sumando opiniones: Antecedentes Históricos y Desarrollos Metodológicos de la Industria de la Opinión Pública en Chile. Documentos de Trabajo ICSO, 3(15).

Escobar, J., y Cuervo, A. (2008). Validez de contenido y juicio de expertos: una aproximación a su utilización. Avances en medición . 6(1), 27-36.

Galicia, L., Balderrama, J., y Edel Navarro, R. (2017). Validez de contenido por juicio de expertos: propuesta de una herramienta virtual. Apertura (Guadalajara, Jal.), 9(2), 42-53. doi: https://dx.doi.org/10.18381/ap.v9n2.993

González, P. (2017). Validación de un instrumento de evaluación de las competencias adquiridas en las prácticas clínicas del grado de enfermería de la UCM. 
(Tesis Doctoral). Facultad de Enfermería, Fisioterapia y Podología. Universidad Complutense. Madrid: Ed. Universidad Complutense.

Guirao-Goris Silamani, J. A., Ferrer Ferrándiz, E., y Montejano Lozoya, R. (2016). Validez de criterio y de constructo del diagnóstico de enfermería "estilo de vida sedentario" en personas mayores de 50 años. Rev. Esp. Salud Publica Recuperado de http://scielo.isciii.es/scielo.php?script=sci_a rttext\&pid=S1135-

57272016000100404\&lng=es. .

Hernández,A., Illesca, M., y Cabezas,M. (2013). Opinión de Estudiantes de la Carrera de Enfermería Universidad Autónoma de Chile, Temuco, Sobre las prácticas clínicas. Ciencia y Enfermería, 19 (1),131-144.

doi:http://dx.doi.org/10.4067/S071795532013000100012.

Huneeus, C. (1999). Las encuestas de opinión pública en las nuevas democracias de América Latina. Contribuciones, XVI( 62), 9-30.

Leyva, Y. (2011). Una reseña sobre la validez de constructo de pruebas referidas a criterio. Perfiles educativos, 33(131), 131154.

Manterola, C., Grande, L. Otzen, T., García, N., Salazar, P., y Quiroz, G. (2018). Confiabilidad, precisión o reproducibilidad de las mediciones. Métodos de valoración, utilidad y aplicaciones en la práctica clínica. Revista chiena de infectología. 35( 6), 680688. doi: http://dx.doi.org/10.4067/S071610182018000600680

Martínez, I. (2008). Encuentas de opinión: de la teoría a la práctica. Metodología de Encuestas 10, 7-25 Gabinete de Prospección Sociológica. Gobierno Vasco

Ortiz, L., y Vergel, M. (2016). Fiabilidad de instrumento para medir resolución de problemas en grado tercero en población vulnerable. Logos Ciencia y

Tecnología, 7 (3), 86-93

Paniagua R. (2015). Metodología

para la validación de una escala o instrumento de medida. Medellín:

Universidad de Antioquia. Facultad Nacional de Salud Pública. Recuperado de http://www.udea.edu.co/wps/wcm/connect/ udea/d76a0609-c62d-4dfb-

83dc5313c2aed2f6/METODOLOG\%C3\%8

DA+PARA+LA+VALIDACI\%C3\%93N+

DE+UNA+ESCALA.pdf?MOD=AJPERES

Real Academia Española.(2020). Diccionario de la lengua española, 23. ${ }^{\text {a }}$ ed., . Recuperado de https://dle.rae.es>

Reguant-Álvarez, M., y TorradoFonseca, M. (2016). El método Delphi. REIRE, Revista d'Innovació i Recerca en Educació, 9 (1), 87-102. doi: 10.1344/reire2016.9.1916

Torrecilla-Salinas, C., De Troyer, O., Escalona, M., y Mejías M. (2017). Una aplicación práctica del método Delphi para la validación de una propuesta de Ingeniería Web Conference Paper. Recuperado de https://www.researchgate.net/publication/31 9108674_Una_aplicacion_practica_del_met odo_Delphi_para_la_validacion_de_una_pr opuesta_de_Ingenieria_Web

Uribe, L., y Rivas, J. (2010). Percepción de los alumnos de enfermería sobre el desempeño docente en la práctica clínica. Enfermería universitaria 7( 4 ), 29-34.

Urrutia Egaña, M., Barrios Araya, S., Gutiérrez Núñez, M., \& Mayorga Camus, M. (2014). Métodos óptimos para determinar validez de contenido. Educación Médica Superior, 28(3), 547-558. Recuperado de http://scielo.sld.cu/scielo.php?script=sci_art text\&pid=S086421412014000300014\&lng=es\&tlng=es.

Von der Gracht, H.A. (2012). Consensus measurement in Delphi studies, 
Revista científica de la Asociación de Historia y Antropología de los Cuidados (Universidad de Alicante)

Technological Forecasting and Social

Change, Elsevier. vol. 79(8), 1525-1536.

\section{ANEXOS}

Anexo 1. Valores para calcular el coeficiente $\mathbf{k}_{\mathbf{a}}$

\begin{tabular}{|l|c|c|c|}
\hline \multirow{2}{*}{ Fuentes de argumentación } & \multicolumn{3}{|c|}{$\begin{array}{c}\text { Grado de influencia de cada una de las } \\
\text { fuentes en sus criterios. }\end{array}$} \\
\cline { 2 - 4 } & A & $\begin{array}{c}\text { M } \\
\text { (Alto) }\end{array}$ & $\begin{array}{c}\text { B } \\
\text { (Bajo) }\end{array}$ \\
\hline Análisis teóricos realizados por usted & 0.3 & 0.2 & 0.1 \\
\hline Su experiencia en el tema & 0.5 & 0.4 & 0.2 \\
\hline Trabajos de autores nacionales consultados & 0.05 & 0.05 & 0.05 \\
\hline Trabajos de autores extranjeros consultados & 0.05 & 0.05 & 0.05 \\
\hline $\begin{array}{l}\text { Su propio conocimiento del estado del } \\
\text { problema en el extranjero }\end{array}$ & 0.05 & 0.05 & 0.05 \\
\hline Su intuición & & & 0.05 \\
\hline
\end{tabular}

Fuente: Elaboración propia 


\begin{tabular}{|c|c|}
\hline Aspectos & $\begin{array}{c}\text { ANEXO } 2 \\
\text { Ítems de Encuesta de opnión de estudiantes } 2018 \text { (incorpora sugerencias de } \\
\text { expertos) }\end{array}$ \\
\hline \multirow{8}{*}{ Generales } & $\begin{array}{l}\text { 1. La información sobre este examen (objetivo y condiciones de realización) fue } \\
\text { entregada anticipadamente por mi escuela. }\end{array}$ \\
\hline & $\begin{array}{l}\text { 2. Las instrucciones que entregaron los examinadores antes del inicio del examen } \\
\text { fueron claras. }\end{array}$ \\
\hline & $\begin{array}{l}\text { 3. El tiempo asignado a la primera parte del examen fue suficiente para } \\
\text { responderlo. }\end{array}$ \\
\hline & $\begin{array}{l}\text { 4. El tiempo asignado a la segunda parte del examen fue suficiente para } \\
\text { responderlo. }\end{array}$ \\
\hline & 5. Las instrucciones del cuadernillo eran claras. \\
\hline & 6. Las preguntas estaban redactadas de forma clara y precisa. \\
\hline & $\begin{array}{l}\text { 7. La cantidad de preguntas por área era adecuada para evaluar los conocimientos } \\
\text { más relevantes que debe tener un egresado de Enfermería. }\end{array}$ \\
\hline & 8. La dificultad de la prueba correspondía al nivel de un egresado de Enfermería. \\
\hline \multirow{4}{*}{$\begin{array}{l}\text { Representatividad } \\
\text { y dominio de las } \\
\text { áreas }\end{array}$} & $\begin{array}{l}\text { 9. El examen abarcó preguntas relevantes de todas las áreas del rol profesional de } \\
\text { enfermería. }\end{array}$ \\
\hline & $\begin{array}{l}\text { 10. Las preguntas del área del Adulto y Adulto Mayor abordaron problemas de salud } \\
\text { de relevancia epidemiológica. }\end{array}$ \\
\hline & $\begin{array}{l}\text { 11. El enfoque de las preguntas del área del Adulto y Adulto Mayor se centró en la } \\
\text { gestión del cuidado de enfermería. }\end{array}$ \\
\hline & $\begin{array}{l}\text { 12. Luego de responder el examen, considero tener dominio teórico de la gestión } \\
\text { del cuidado en el área del Adulto y Adulto Mayor. }\end{array}$ \\
\hline
\end{tabular}


13. Las preguntas del área del Recién Nacido, Niño/Niña y Adolescente, abordaron problemas de salud de relevancia epidemiológica.

14. El enfoque de las preguntas del área del Recién Nacido, Niño/Niña y Adolescente, se centró en la gestión del cuidado de enfermería.

15. Luego de responder el examen, considero tener dominio teórico de la gestión del cuidado en el área del Recién Nacido, Niño/Niña y Adolescente.

16. Las preguntas del área de Gestión y Liderazgo eran representativas del quehacer profesional de enfermería

17. Luego de responder el examen, considero tener dominio teórico en el área de Gestión y Liderazgo.

18. Las preguntas del área de la Disciplina y Profesión eran representativas del quehacer profesional de enfermería.

19. Luego de responder el examen, considero tener dominio teórico en el área de la Disciplina y de la Profesión.

Utilidad examen del

20. Este examen es útil para evaluar los conocimientos de los egresados en áreas establecidas del quehacer profesional.

21. El hecho de que el examen sea voluntario influyó en mi dedicación al responderlo.

22. Este examen debería ser obligatorio para todos los egresados de enfermería.

23. La rendición de este examen debe ser un requisito para comenzar a ejercer como profesional. 